\title{
Functional Characterization of Muscarinic Receptors in Human Schwann Cells
}

\author{
Roberta Piovesana ${ }^{1,2}$, Alessandro Faroni ${ }^{2}\left(\mathbb{D}\right.$, Ada Maria Tata ${ }^{1,3, *(\mathbb{D})}$ and Adam J. Reid $2,4, *(\mathbb{D})$ \\ 1 Department of Biology and Biotechnology "C. Darwin”, Sapienza University of Rome, P.le Aldo Moro, 5, \\ 00185 Rome, Italy; roberta.piovesana@uniroma1.it \\ 2 Blond McIndoe Laboratories, Division of Cell Matrix Biology and Regenerative Medicine, \\ School of Biological Sciences, Faculty of Biology, Medicine and Health, The University of Manchester, \\ Manchester Academic Health Science Centre, Manchester M13 9PT, UK; alessandro.faroni@manchester.ac.uk \\ 3 Research Centre of Neurobiology "Daniel Bovet”, Sapienza University of Rome, P.le Aldo Moro, 5, \\ 00185 Rome, Italy \\ 4 Department of Plastic Surgery \& Burns, Wythenshawe Hospital, Manchester University NHS Foundation \\ Trust, Manchester Academic Health Science Centre, Manchester M23 9LT, UK \\ * Correspondence: adamaria.tata@uniroma1.it (A.M.T.); adam.reid@manchester.ac.uk (A.J.R.); \\ Tel.: +39-06-49912822 (A.M.T.); +44-161-275-1596 (A.J.R.)
}

Received: 23 July 2020; Accepted: 9 September 2020; Published: 11 September 2020

\begin{abstract}
Functional characterization of muscarinic cholinergic receptors in myelinating glial cells has been well described both in central and peripheral nervous system. Rat Schwann cells (SCs) express different muscarinic receptor subtypes with the prevalence of the M2 subtype. The selective stimulation of this receptor subtype inhibits SC proliferation, improving their differentiation towards myelinating phenotype. In this work, we describe for the first time that human SCs are cholinoceptive as they express several muscarinic receptor subtypes and, as for rat SCs, M2 receptor is one of the most abundant. Human SCs, isolated from adult nerves, were cultured in vitro and stimulated with M2 muscarinic agonist arecaidine propargyl ester (APE). Similarly to that observed in rat, M2 receptor activation causes a decreased cell proliferation and promotes SC differentiation as suggested by increased Egr2 expression with an improved spindle-like shape cell morphology. Conversely, the non-selective stimulation of muscarinic receptors appears to promote cell proliferation with a reduction of SC average cell diameter. The data obtained demonstrate that human SCs are cholinoceptive and that human cultured SCs may represent an interesting tool to understand their physiology and increase the knowledge on how the cholinergic stimulation may contribute to address human SC development in normal and pathological conditions.
\end{abstract}

Keywords: human Schwann cells; acetylcholine; muscarinic receptors; differentiation; glial cells

\section{Introduction}

During development, the close relationship between neurons and Schwann cells (SCs), the main glial cell population in peripheral nervous system (PNS), is mediated by different molecules including acetylcholine (ACh) [1-5]. In particular, in nerve fibres of invertebrates, ACh receptors on SCs localize to the axon-SCs boundary [6]. Myelinating glia cells express both muscarinic and nicotinic ACh receptor subtypes during development and in adulthood [1]. Several studies demonstrated that ACh signalling, via muscarinic receptor activation, is able to modulate proliferation and differentiation in astrocytes [7] and oligodendrocytes [8].

Cultures of SCs, obtained from sciatic nerve of 2 days post-natal rats express muscarinic receptors (M1-M4) [9]. In particular, M2 mAChR subtype is the most abundant and persists in mature SCs [10]. 
SC treatment with cholinergic agonists, triggers significant changes in intracellular inositol 1,4,5-triphosphate (IP3) and cyclic adenosine monophosphate (cAMP) levels [9]. Cells treated with a selective agonist of M2 muscarinic subtype arecaidine propargyl ester (APE) showed an arrest of SC proliferation in the G1-S phase [11] supported by the negative modulation of c-Jun and Notch-1, thereby reducing SC proliferation [10]. M2 receptor stimulation promotes SC differentiation towards myelinating phenotype with the upregulation of promyelinating transcription factors as Sox10 and Egr2, followed by an increased expression of myelin proteins (e.g., myelin basic protein, MBP; myelin protein zero, $\mathrm{P0}$ and peripheral myelin protein 22, PMP22) [10]. Ultrastructural analyses by transmission electron microscopy (TEM) of the sciatic nerves of M2/M4 knock-out mice have demonstrated the presence of degenerating axons and alterations in myelin organization of the medium/large axons [10]. Moreover, cholinergic stimulation, but most evidently the activation of M2 receptor, modulates nerve growth factor (NGF) levels, negatively regulating the proNGF-B isoform involved in apoptosis [12].

These results clearly demonstrate that, at least in the rat model, M2 mAChRs negatively modulate proliferation and upregulate promyelinating transcriptional factors and myelin protein expression, promoting SC differentiation.

It is relevant to note that human SCs (hSCs) have been poorly investigated. Increasing knowledge on hSCs in this field is of relevance, considering the role of SCs in different peripheral pathologies including neurofibromatosis and peripheral neuropathies [13-15]. Starting from the described role of M2 receptor in rat SC physiology, in the present study we have analysed cultures of hSCs derived from sensory nerves withdrawn from human adipose tissue. As observed in the rat model, cholinergic receptors are expressed also in hSCs. The preferential activation of M2 receptor negatively modulates hSC proliferation without affecting cell survival and supporting cell differentiation. Instead, the non-selective muscarinic receptors stimulation appears to promote cell proliferation with a reduction of the average cell diameter. These data highlight that hSCs, similarly to what observed in rat SCs, are cholinoceptive and cholinergic stimulation may be involved in hSC physiology without affecting their plasticity.

\section{Results}

\subsection{Muscarinic Receptors Are Expressed in hSCs}

Human SCs, obtained from 3 different patients, were first characterized for the expression of S100 $\beta$, as SC marker. As showed in Figure 1A, the cells presented a more pronounced spindle-like morphology, and the $90 \%$ of the cells in cultures resulted immune-positives for S100 $\beta$.

Then, the expression of cholinergic muscarinic receptor subtypes was evaluated by RT-PCR analysis. As reported in Figure 1B, in hSCs from 3 different patients, M1, M2, and M3 subtypes were expressed at higher levels, whereas M4 and M5 expression appeared to be variable between different patients. Similar results were obtained by qRT-PCR analysis (Figure S1). M2 subtype transcripts were present in all patients, and its expression at protein level has been confirmed by Western blot analysis. As shown in Figure 1C, M2 muscarinic subtype was expressed in all patients albeit at variable levels and with evident glycosylation pattern of the receptors between different patients. Cell cultures obtained from these three patients were stimulated for 3 days in vitro with M2 agonist APE; this agonist has been largely characterized in different murine and human cell lines where its ability to specifically bind M2 receptor subtype was largely demonstrated $[10,16,17]$. As reported in Figure 1D, M2 stimulation with $100 \mu \mathrm{M}$ APE resulted in a significant decrease of cell growth in all patients after 3 days of treatment. 
A
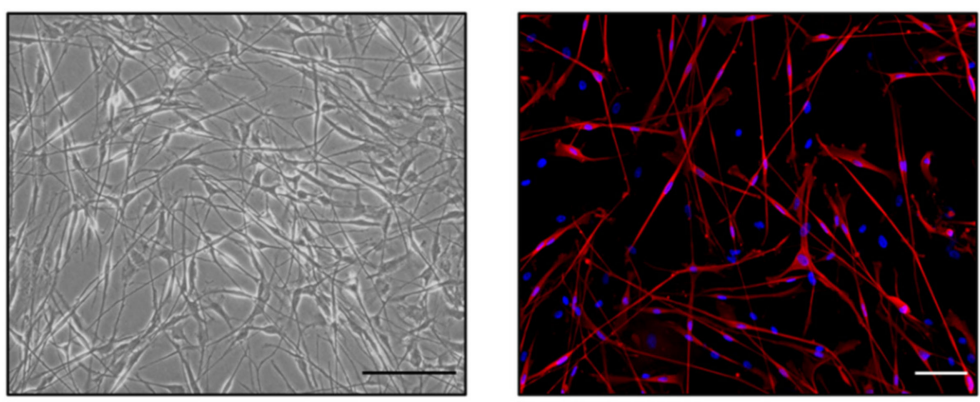

S100ß DAPI

B

P1

P2

P3

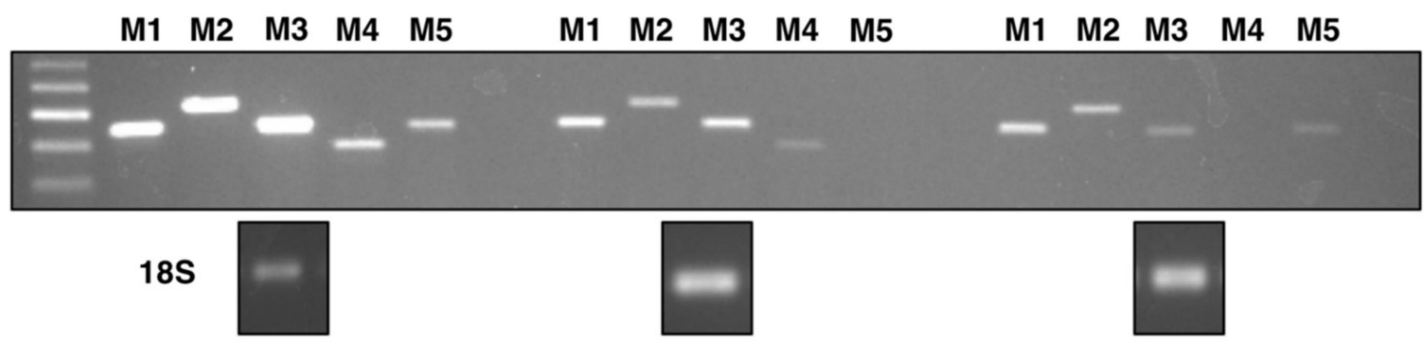

C

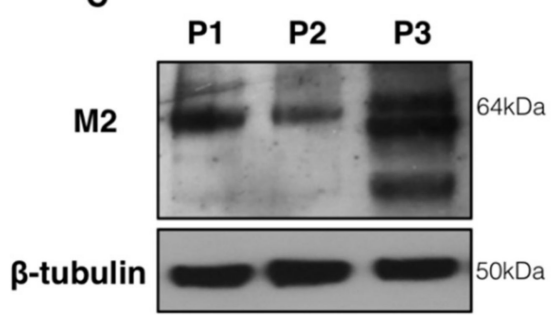

D

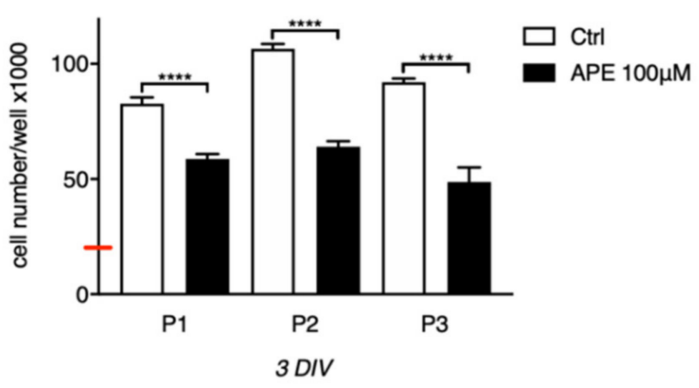

Figure 1. (A) Human Schwann cells (hSCs) were photographed at contrast-phase microscope (scale bar $=100 \mu \mathrm{m}$ ); hSCs show a typical bipolar morphology. Immunostaining for S100 $\beta$ appeared present in the $90 \%$ of the cultured cells (scale bar $=100 \mu \mathrm{m}$ ). (B) Analysis by RT-PCR of muscarinic receptors subtypes in hSCs obtained from 3 different patients (P1, P2, and P3). Here, 18s was used as housekeeping gene. (C) M2 muscarinic receptor expression of the protein samples obtained from the same patients analysed by RT-PCR. $\beta$-tubulin was used as reference protein. (D) MTS assay showing the ability of M2 agonist APE to reduce cell growth in all three patients. The results are the average \pm SEM of three independent experiments performed in triplicate $(* * * p<0.0001)$. The red bar represents the number of cells plated $\left(20 \times 10^{3}\right.$ cells/well). The day after plating, cells were treated with $100 \mu \mathrm{M}$ APE and MTS assay was performed after 3 days of treatment.

\subsection{Analysis of Cell Growth, Survival, and Morphology}

In order to evaluate the ability of muscarinic receptors to modulate hSC development, we analysed the cell growth by MTS assay for up to 7 days of $100 \mu \mathrm{M}$ APE or muscarine treatments in more patients $(n=5)$ (Figure 2A). APE treatment decreased cell growth after $72 \mathrm{~h}$ of treatment, remaining substantially lower if compared with untreated cells at 7 days of treatment. Instead, the non-selective agonist muscarine, used at the same final concentration of $100 \mu \mathrm{M}$, promoted cell growth after 5 days of treatment in vitro (DIV), albeit an initial decrease of cell number after 3 days of treatment was evident (Figure 2A). Statistical analysis between different time points, reported in the Supplementary Materials, showed that although a significant increase of cell growth between different time points (e.g., APE 
3 DIV vs. APE 7 DIV) was observed, cell growth decreased between APE treatment and untreated cells at every time point (Table S1). Considering this apparent increase of cell growth upon 7 days of $100 \mu \mathrm{M}$ APE treatment, in order to evaluate if the effect may be dependent on reduced activity of M2 agonist during 7 days in vitro, we performed the same experiment at different concentrations of APE, modifying the experimental plan with the media change with or without APE treatment at the third day of treatment. In this experimental condition, differently to what was observed in the previous experiment reported in Figure 2A, we observed that the cell growth was unchanged at 3 DIV and 7 DIV after $100 \mu \mathrm{M}$ APE treatment, confirming the inhibitory effect of the high dose of APE on cell growth. Moreover, the results obtained indicated that only APE at concentrations of 50 and $100 \mu \mathrm{M}$ was able to significantly reduce the cell growth but the $50 \mu \mathrm{M}$ APE effect was evident only after 7 days of treatment, whereas lower concentrations $(25 \mu \mathrm{M})$ did not show any effects (Figure 2B). Similarly, the analysis of cell growth at different concentrations of muscarine (ranging from 25 to $100 \mu \mathrm{M}$ ) demonstrated that the low doses of the non-selective agonist did not show any effects on cell growth (data unpublished) and that only the concentration of $100 \mu \mathrm{M}$ was able to positively modulate cell proliferation (Figure 2A).

A

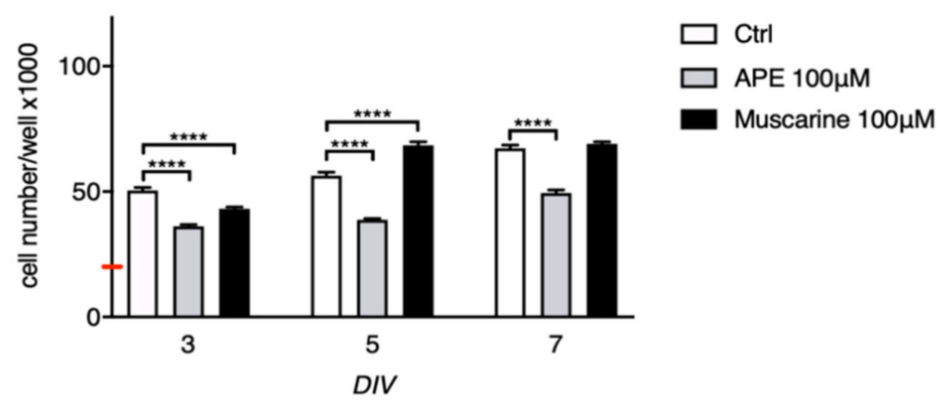

B

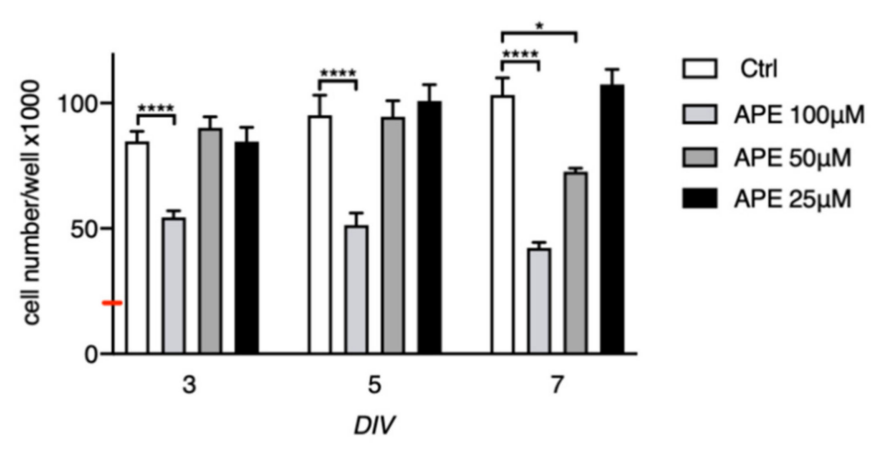

Figure 2. MTS assay in hSCs maintained up to 7 days of treatment in vitro (DIV) in presence or absence of muscarinic agonists. (A) APE $(100 \mu \mathrm{M})$ exposure was able to decrease cell growth up to 7 days of treatment $(* * * * p<0.0001$; APE vs. ctrl at the same time point). The treatment with $100 \mu \mathrm{M}$ muscarine appeared to promote cell proliferation after 5 days of treatment ${ }^{* * * *} p<0.0001$; muscarine vs. ctrl at the same time point). Data are represented as mean \pm SEM of three independent experiments performed in triplicate in five different patients. One-way ANOVA with Tukey's post hoc test between different time points is reported in the Supplementary Materials. (B) hSCs cell growth was also evaluated at different APE concentrations $(25,50$, and $100 \mu \mathrm{M})$ at 3, 5, and 7 DIV. ANOVA with Dunnett multiple comparison analyses was performed against control at the same time point. Data are represented as mean \pm SEM of three independent experiments performed in triplicate in five different patients $(* * * * p<0.0001$; $\left.{ }^{*} p<0.05\right)$. The red bar represents the number of cells plated $\left(20 \times 10^{3}\right.$ cells/well). The day after plating, cells were treated with both muscarinic agonists at the final concentration of $100 \mu \mathrm{M}(\mathrm{A})$ or with different concentrations of APE (B); MTS assays were performed after 3, 5, and 7 days of treatment. 
The cholinergic treatments were not toxic for hSCs; trypan blue staining was used to determine possible drug toxicity; both agonists did not show toxic effects on hSC vitality at $24 \mathrm{~h}$ of treatment (Figure 3A-D). Morphological analysis, performed using Cell Countess, showed a significant increase of the average cell diameter after M2 stimulation (Figure 3F,H). Conversely, muscarine showed an opposite effect, reducing the average cell diameter of the cells (Figure 3G,H). Moreover, APE treatment caused a lower density of cells compared to the untreated cells (Figure 3I,J), supporting the reduction of cell growth observed by MTS assay. Interestingly, hSCs treated with APE also showed a more elongated morphology (Figure 3J). This result was supported by a significant upregulation of Egr2 protein levels, a typical promyelinating transcriptional factor, after M2 agonist exposure (Figure 3L,M).

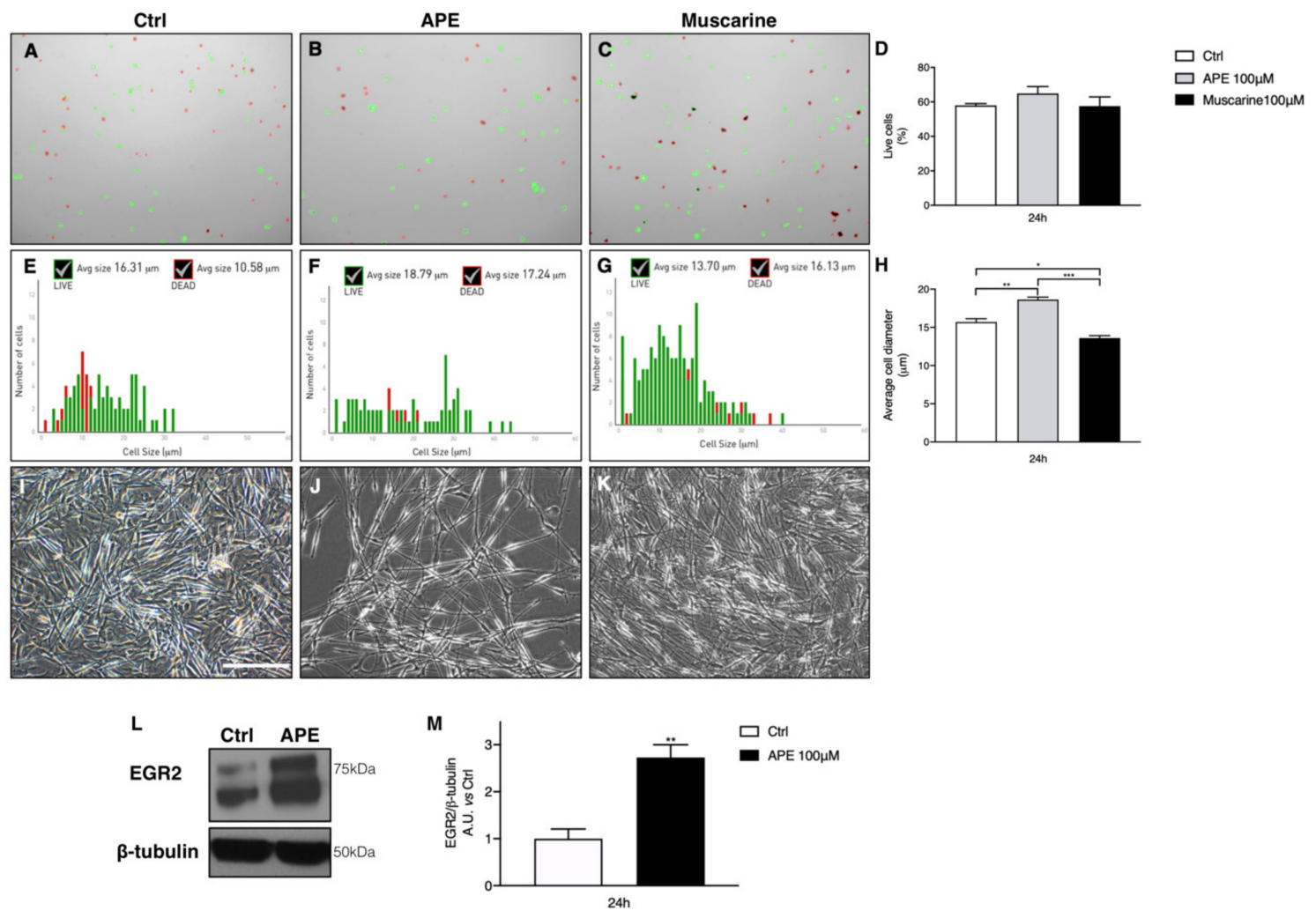

Figure 3. Analysis of cell death after trypan blue assay, using Cell Countess. (A-D) The analysis showed that both cholinergic agonists are not toxic for hSCs $((\mathbf{A}-\mathbf{C})$, green dots are live cells, whereas red dots are dead cells). In (D), the percentage of the live cells under different experimental conditions is reported. (E-G) Countess analysis shows a significant increase of average cell diameter. The graphs show the analysis of cell diameter, where the green columns and the red columns represent the live cells or dead cells, respectively, presenting a specific diameter. Up the panels, the analyses show the average cell diameter for live and dead cells ((E) control; (F) APE; (G) muscarine)). In (H), the average of cell diameter is reported; APE induces an increase of cell diameter after $24 \mathrm{~h}$ of treatment ${ }^{* *} p<0.01$ APE vs. ctrl; $\mathrm{APE}=18.63 \pm 0.33 \mu \mathrm{m} ;{ }^{* * *} p<0.01$ APE vs. Muscarine), whereas muscarine exposure induces a reduction in average cell diameter $\left({ }^{*} p<0.05\right.$ muscarine vs. ctrl; muscarine: $\left.13.60 \pm 0.32 \mu \mathrm{m}\right)$. (I-K) Images in phase contrast showing hSC morphology and density after APE and muscarine treatments (scale bar: $100 \mu \mathrm{m}) ;((\mathbf{I})$ control; (J) APE; (K) muscarine)). (L,M) The analysis of Egr2 protein expression, by Western blot analysis, has shown that APE induces a significant upregulation of Egr2 protein expression $(* * p<0.01$, APE treatment vs. $\operatorname{ctrl}=2.73 \pm 0.27)$. $\beta$-tubulin was used as protein reference. Data are represented as mean \pm SEM of three independent experiments performed in three different patients. 


\section{Discussion}

During development and peripheral nerve injury, SCs produce and secrete a variety of neurotrophic factors and extracellular matrix components that provide a helpful microenvironment for neuronal survival and axon elongation [18]. Transplanted SCs can improve nerve regeneration and then myelinate the regenerated axons, although their isolation is difficult and requires healthy nerve sacrifice.

Our knowledge on SC development and plasticity is derived mainly from rodent models (rat and mice), whereas hSC s have been poorly investigated. Understanding hSC physiology could be relevant for the improvement of therapeutic interventions in peripheral pathologies such as neuropathies and in peripheral nerve injury (PNIs).

In the rat model, CNS and PNS myelinating glia are cholinoceptive and express both muscarinic and nicotinic cholinergic receptors at different times of development [7-11]. Moreover, also after nerve injury, ACh plays a significant role in motor nerve terminal outgrowth and muscle repair. In fact, the inhibition of postsynaptic neuromuscular ACh receptors by $\alpha$-bungarotoxin $(\alpha$-Btx) in the skeletal muscles significantly reduces nerve outgrowth [19], suggesting that a local release of ACh is essential to directly trigger nerve terminal outgrowth [3].

Cultures of rat immature SCs express muscarinic receptor (M1-M4), with a higher expression of M2 mAChR subtype, which increases the expression of myelin proteins [9,10], addressing SCs towards promyelinating phenotype. ACh secreted by the nerve terminal can also modulate perisynaptic SCs (PSCs), which surround nerve terminals at the neuromuscular junction, and regulates glial fibrillary acidic protein (GFAP) expression and cytoskeletal changes via mAChRs [20].

In the present work, for the first time, we demonstrate that hSCs are cholinoceptive; in fact, they express several muscarinic receptor subtypes, albeit the expression levels of the single subtypes are variable between different patients. Similarly to that observed in rat, they express M2 muscarinic receptor that is also differentially expressed in different patients. However, M2 agonist, APE, is able to decrease SC growth without affecting cell viability, as indicated by trypan blue staining. In particular, $100 \mu \mathrm{M}$ APE is able to arrest cell growth during 7 DIV. Moreover, we have observed that $50 \mu \mathrm{M}$ APE is also able to decrease cell growth only after 7 DIV. This may be dependent on distinct reasons: 1. the affinity of the M2 receptor for APE may vary according to the glycosylation grade of the receptors in the different patients, as observed in Western blot analysis and 2. the prolonged exposure to $50 \mu \mathrm{M}$ APE may be necessary to activate the M2 downstream signalling transduction pathway.

According to their physiological role, SCs change their morphology, being much longer when they are induced towards a myelinating phenotype [21]. Morphological analyses show that hSCs cultured in growth condition, in presence of forskolin (Fsk) and glial growth factor 2 (GGF-2) in the culture media, have a classic bipolar morphology. According to our previous data gathered in the rat model [10,11], APE treatment elongates hSCs with a significant increase of the average cell diameter and Egr2 protein expression, suggesting that the M2 selective stimulation enhances promyelinating phenotype also in hSCs [10]. The ability of muscarinic receptors to modulate Egr2 transcription factor family is well documented [22,23]. Interestingly the modulation of Egr2 by M2 receptors may at least explain the inhibitory effect on cell proliferation. SC proliferation is in fact supported by Neuregulin 1 (NRG1) pathway and c-jun expression. Our previous studies in rat have largely demonstrated the ability of M2 receptors to downregulate cyclic adenosine monophosphate (cAMP) levels and c-jun expression [9,17]. It is possible to hypothesize that the same mechanism was maintained in hSCs. Moreover c-jun and Egr2 have a cross-antagonistic relationship [24], thus the upregulation of Egr2-M2 mediated, may indirectly contribute to a decrease of c-jun expression and subsequent SC proliferation.

In the present study, it has not been possible to perform pharmacological competition studies with selective antagonists for muscarinic receptors considering the limited number of the cells obtained from the same patients. Albeit the APE-derived compounds were reported as preferential M1-M4 agonists [25], several structural variations of the molecules appeared to improve the selectivity for M2 receptor [26,27]. In particular, the M2 agonist APE has been largely characterized by our group by pharmacological competition studies performed in rat glial cells and DRG sensory neurons, clearly 
demonstrating that APE effects are largely counteracted only by gallamine, one of the preferential M2 receptor antagonist [8,28]. Moreover, in neuroblastoma and glioblastoma cell lines, we have also demonstrated that M1 and M3 antagonists, pirenzepine and 4-DAMP, respectively, were not able to counteract APE effects, confirming preferential selectivity of APE for M2 receptor. Moreover, after M2 knockdown by short interference RNA, APE effect was completely abolished [16,29-31]. Finally, it is relevant to note that M4 receptor expression both in rat [9] and in hSCs appears significantly lower than M2 subtype, at least at transcript level; for this reason, the potential interference of M4 receptors, should be irrelevant.

Muscarine treatment, instead, promotes cell proliferation, whereas the cell morphology after non-selective agonist treatment, remains similar to untreated cells, albeit the reduction of cell diameter observed. The contrasting results obtained with muscarine are likely dependent on the ability of muscarine to bind all muscarinic receptor subtypes. The positive effects of muscarine on cell proliferation may be dependent on the simultaneous activation of Gq11-proteins, probably M1- and M3-mediated ones. The reduction of the cell size may be dependent on more intense SC proliferation after muscarine treatment. In fact, when the cells are in proliferation, they assume a rounded morphology, and after the mitotic cell division, they have a reduced dimension.

\section{Materials and Methods}

\subsection{Human Schwann Cells Cultures}

Human nerves were isolated from adipose tissue of patients undergoing reconstructive surgery at Wythenshawe Hospital, Manchester University NHS Foundation Trust, UK, after informed consent was granted. All patients were female, healthy, and aged 44-64 years. All procedures were approved by the National Research Ethics Committee, UK (NRES 13/SC/0499) and conformed with the World Medical Association Declaration of Helsinki.

Human nerves were dissected and immersed into high-glucose DMEM (Sigma-Aldrich, St. Louis, MO, USA) containing 10\% (v/v) foetal bovine serum (FBS, LabTech, Uckfield, UK), $2 \mathrm{mM}$ L-glutamine (GE Healthcare, Chicago, IL, USA), and $1 \%(v / v)$ penicillin-streptomycin solution (SC media). Connective tissue was removed with tweezers and the single fibres extracted were cut in small pieces (approximately $1 \mathrm{~mm}$ each) and cultured in a $60 \mathrm{~cm}^{2}$ dish with SC media supplemented with $10 \mu \mathrm{M}$ forskolin (Fsk; Sigma-Aldrich) $+100 \mathrm{ng} / \mathrm{mL}$ glial growth factor 2 (GGF-2, Acorda Therapeutics, Ardsley, NY, USA) for 2 weeks. Media were changed every 3 days. After 2 weeks of culture, human nerves were digested with Dispase (Life Technologies, Carlsbad, CA, USA)/Collagenase IV (Life Technologies), in a ratio of 1:1, for $24 \mathrm{~h}$. The day after the solution containing nerves partially digested was collected in $15 \mathrm{~mL}$ falcon tube and nerves were gently triturated using a glass pipette. Cell suspension was passed through a sterile $70 \mu \mathrm{m}$ mesh to remove clumps of undissociated tissue and axon debris and then centrifuged at $900 \mathrm{rpm}$ for $5 \mathrm{~min}$. Cell pellet was gently resuspended in SC media supplemented with $10 \mu \mathrm{M}$ forskolin (Fsk, Sigma-Aldrich) $+100 \mathrm{ng} / \mathrm{mL}$ glial growth factor 2 (GGF-2, Acorda Therapeutics, Ardsley, NY, USA) and plated in a T75 flask. T75 flask was previously coating with Poly-D-Lysine (Sigma-Aldrich, P7280) for $30 \mathrm{~min}$ at RT and subsequently coating with laminin $\left(10 \mathrm{mg} / \mathrm{mL}\right.$, Sigma-Aldrich) for $2-3 \mathrm{~h}$ at $37^{\circ} \mathrm{C}$. Cells were maintained in T75 flasks at $37^{\circ} \mathrm{C}$ and $5 \% \mathrm{CO}_{2}$, with three medium changes every week.

\subsection{Pharmacological Treatments}

Human SCs were treated with M2 selective agonist arecaidine propargyl ester hydrobromide (APE, A140; Sigma-Aldrich) at the final concentration ranging from 25 to $100 \mu \mathrm{M}$. Albeit APE was reported as preferential M1-M4 agonist [25], several modifications of the molecule suggested a preferential agonistic effect on M2 receptor subtype [26,27]. Our works have been focused on the characterization of APE hydrobromide by pharmacological competition studies with M2 antagonists methoctramine or gallamine $[8,17,32]$. Moreover, the silencing of M2 receptors by siRNAs have largely demonstrated 
the selectivity of APE for M2 receptor in several cell lines (i.e., human glioblastoma, neuroblastoma, and urothelial bladder cell lines [16,29-31,33].

To study all muscarinic receptors activation, hSCs were treated with the non-selective muscarinic agonist, muscarine (Sigma-Aldrich), at the final concentration ranging from 25 to $100 \mu \mathrm{M}$.

All experiments were performed in technical and experimental triplicate.

\subsection{Cell Proliferation and Survival Assay}

Human SCs from different patients were plated in 24-well plates (Corning Life Science, Amsterdam, Netherlands) at a density of $20 \times 10^{3}$ cells/well. The day after plating, cells were treated with both muscarinic agonists, APE and muscarine, as mentioned above. Cell proliferation was evaluated at 3,5 , and 7 days of treatment. Cell medium was aspirated, and cells were incubated in 20\% (v/v) CellTiter 96 AQueous One Solution Cell Proliferation Assay, [3-(4,5-dimethylthiazol2-yl)-5-(3-carboxymethoxyphenyl)-2-(4-sulfophenyl)-2H-tetrazolium, inner salt; MTS; Promega, Southampton, UK), diluted in phenol-free DMEM (Sigma-Aldrich). Following $2 \mathrm{~h}$ of incubation at $37^{\circ} \mathrm{C}$, the absorbance at $490 \mathrm{~nm}$ was recorded with an Asys UVM-340 microplate reader/spectrophotometer (Biochrom, Cambridge, UK). After the standard curve was performed, data were expressed as cell number \pm SEM.

Under the same experimental conditions, we evaluated the presence of dead cells. After trypsinization, the percentage of dead cells was read with Cell Countess (Thermo Fisher Scientific, Waltham, MA, USA), using trypan blue staining (1:10 $v / v$, Thermo Fisher Scientific, Altrincham, UK). Cell Countess analysed live (green) and dead (red) cells (as shown in Figure 3A-C); moreover, Cell Countess reported every analysed diameter in a graph (Figure $3 \mathrm{E}-\mathrm{G}$ ) in which the green columns represent the live cell diameter and the red columns represent the dead cell diameter. The results were reported in a column graph, and ANOVA analysis followed by Tukey post hoc test was performed.

\subsection{RNA Extraction and RT-PCR Analysis}

Cells were collected and stored in RNA cell protect agent (Qiagen, Hilden, Germany). Total RNA was isolated from hSCs using RNeasy Plus Mini Kit (Qiagen), according to the manufacturer's protocol. RNA was quantified using a NanoDrop ND-100 spectrophotometer (Thermo Fisher Scientific). Each sample was reverse-transcribed using RT2 First Strand Kit (Qiagen), according to the manufacturer's instructions. RT-PCR was performed with GoTaq Green Master Mix (Promega) using MultiGene Optimax (Labnet, Edison, NJ, USA). The sequences of the used primers are reported in Table 1. Moreover, $18 \mathrm{~s}$ was used as housekeeping gene.

Table 1. List of RT-PCR primers.

\begin{tabular}{ccc}
\hline Gene & Forward $\mathbf{5}^{\prime} \mathbf{-} \mathbf{3}^{\prime}$ & Reverse $\mathbf{5}^{\prime} \mathbf{- \mathbf { 3 } ^ { \prime }}$ \\
\hline RN18S1 & ATCGGGGATTGCAATTATTC & CTCACTAAACCATCCAATCG \\
M1 & CAGCAGTACCGAACCACGTA & CTCCTGACTTCCTGCCTAAA \\
M2 & CCAAGACCCCGTTTCTCCAAG & CCTTCTCCTCTCCCTGAACAC \\
M3 & CGCTCCAACAGGAGGAAGTA & GGAGTTGAGGATGGTGCTGT \\
M4 & AATGAAGCAGAGCGTCAAGAA & TCATTGGAAGTGTCCTTATCA \\
M5 & CCTGGCTGATCTCCTTCATC & GTCCTTGGTTCGCTTCTCTG \\
\hline
\end{tabular}

\subsection{Protein Extraction and Western Blot}

Whole-cells lysates were obtained by scraping cells from confluent 6 well-plates using RIPA Buffer (Sigma-Aldrich) supplemented with protease and phosphatase inhibitors (Thermo Scientific). Lysates were incubated for $30 \mathrm{~min}$ on ice and later centrifuged for $20 \mathrm{~min}$ at $14,000 \mathrm{rpm}$ at $4{ }^{\circ} \mathrm{C}$. According to the manufacturer's protocol, protein concentrations were determined using Pierce ${ }^{\mathrm{TM}} \mathrm{BCA}$ Protein Assay Kit (Thermo Fisher Scientific). Sample buffer $(6 \times)$ was added to protein lysates and heated for 5 min at $100{ }^{\circ} \mathrm{C}$. 
Precisely, $30 \mu \mathrm{g}$ of each sample was loaded onto a 10\% SDS (Sodium dodecyl sulphate) polyacrylamide gel and run at $120 \mathrm{~V}$ using running buffer $(25 \mathrm{mM}$ Tris, $190 \mathrm{mM}$ glycine, $0.08 \%$ $(w / v)$ SDS). SDS-PAGE gels were transferred for $60 \mathrm{~min}$ onto nitrocellulose blotting membranes (GE Healthcare Life Science) at $80 \mathrm{~V}$ in transfer buffer (25 mM Tris-base; $192 \mathrm{nM}$ glycine, 20\% (v/v) methanol). After transfer, membranes were blocked for $1 \mathrm{~h}$ in blocking buffer (Tris-buffer saline (TBS)-Tween solution containing 5\% non-fat dry milk). Membranes were incubated with the primary antibody, diluted in blocking buffer, overnight at $4{ }^{\circ} \mathrm{C}$.

Primary antibodies used were: mouse anti-M2 antibody (1:500, Abcam, Cambridge, UK) and rabbit anti-Egr2 (1:500, ProteinTech, Manchester, UK). After overnight incubation, membranes were washed 5 times with TBS-Tween buffer and incubated for $1 \mathrm{~h}$ at RT with secondary antibody: anti-rabbit horseradish peroxidase (1:2000, Cell Signaling, Hitchin, UK) or anti-mouse horseradish peroxidase (1:1000, Cell Signaling, Hitchin, UK) for chemiluminescence detection. To determine housekeeping protein, membranes were stripped before reblotting with another primary antibody. $\beta$-tubulin was used as protein reference (rabbit anti- $\beta$-tubulin, 1:1000, Abcam, Cambridge, UK).

Membranes were exposed to SuperSignal West Pico Chemiluminescent Substrate (Thermo Fisher Scientific) for signal detection. The bands were detected by exposition to ChemiDoc (Molecular Imager ChemiDoc XRS + System with Image Lab Software, Bio-Rad, CA, USA). The optical density (OD) of each protein band was analysed with ImageJ software (National Institutes of Health, NIH, 469 Bethesda, MD, USA) and normalized against the OD of the protein reference band.

\subsection{Immunocytochemistry Analysis}

After fixation with $4 \%$ paraformaldehyde (PFA, Sigma-Aldrich), cells were incubated with Triton X-100 0.2\% for $30 \mathrm{~min}$ at RT. Then, cells were washed twice with PBS and treated for $1 \mathrm{~h}$ at RT with block solution containing PBS $+0.1 \%$ Triton X-100 and, according with secondary antibody, $10 \%$ normal donkey serum (NDS). Cells were incubated with primary antibody (polyclonal anti-S100 $\beta$, Dako, Glostrup, Denmark) in $0.1 \%$ Triton X-100, 0.1\% (w/v) BSA, and $0.1 \%(w / v)$ sodium azide in PBS at $4{ }^{\circ} \mathrm{C}$ overnight. The day after, cells were washed with PBS three times for $10 \mathrm{~min}$ and incubated with secondary antibody (Donkey anti-rabbit IgG $(\mathrm{H}+\mathrm{L})$ Highly Cross-Adsorbed Secondary Antibody, Alexa Fluor 568, Life Technologies) in $0.1 \%$ Triton X-100, 0.1\% (w/v) BSA, and 0.1\% (w/v) sodium azide in PBS for $1 \mathrm{~h}$ at RT. Then, cells were washed 3 times with PBS and mounted with Vectashield mounting medium for fluorescence containing $4^{\prime}-6^{\prime}$-diamidino-2-phenylindole for nuclear staining (H1200, Vector Lab, DBA, Milan, Italy). Images were taken using a fluorescence microscope (Olympus IX51, Southend-on-Sea, UK).

\subsection{Data Analysis}

Data analyses were performed with GraphPad Prism 8 (GraphPad Software, La Jolla, CA, USA). Data were presented as the mean \pm standard error of the mean (SEM). Student's $t$-test or one-way ANOVA analyses followed by Tukey or Dunnett post hoc tests were used. A value of $p<0.05$ was considered statistically significant: ${ }^{*} p<0.05$, ${ }^{* *} p<0.01,{ }^{* * *} p<0.001$, and ${ }^{* * * *} p<0.0001$. The densitometric analysis of Western blot was performed by ImageJ software (National Institutes of Health, NIH, 469 Bethesda, MD, USA).

\section{Conclusions}

The data obtained in the present work represent the first evidence that hSCs can be isolated by peripheral nerves present in human subcutaneous adipose tissue. Moreover, we demonstrate that they are cholinoceptive and that $\mathrm{ACh}$, via muscarinic receptors, may differently contribute to axon-SCs cross-talk also in human. These results may be of great relevance in future studies to design new therapeutic intervention for the treatment of traumatic peripheral nerve injuries or for peripheral nervous system pathologies such as neurofibromatosis and the peripheral neuropathies. 
Supplementary Materials: The following are available online at http://www.mdpi.com/1422-0067/21/18/6666/s1. Figure S1. qRT-PCR analysis for muscarinic receptor subtypes. Table S1. Statistical analysis of MTS assay after $\mathrm{APE}$ and muscarine treatment at the final concentration of $100 \mu \mathrm{M}$.

Author Contributions: Conceptualization, R.P. and A.M.T.; methodology and acquisition of data R.P.; nerve dissection and cell preparation, A.J.R., A.F., and R.P.; formal analysis, R.P., interpretation of data: R.P., and A.M.T.; writing—original draft preparation, R.P. and A.M.T.; writing—review and editing, R.P., A.M.T. and A.J.R.; All authors have read and agreed to the published version of the manuscript.

Funding: A.F. and A.J.R. are supported by the Hargreaves and Ball Trust, the Academy of Medical Sciences (AMS-SGCL7) and by Seed Corn Funding from the Rosetrees Trust and the Stoneygate Trust (M746). This work was also supported by Ateneo Sapienza Funds 2017 (RM11715C7F959CA4) to A.M.T. and "Avvio Giovani" Project 2018 (AR11816435A0F81D) from Ateneo Sapienza to R.P.; R.P. was also supported by CIB 2018.

Acknowledgments: The authors are grateful to Acorda Therapeutics for kindly supplying us with recombinant GGF-2 for use in this study.

Conflicts of Interest: The authors declare no conflict of interest.

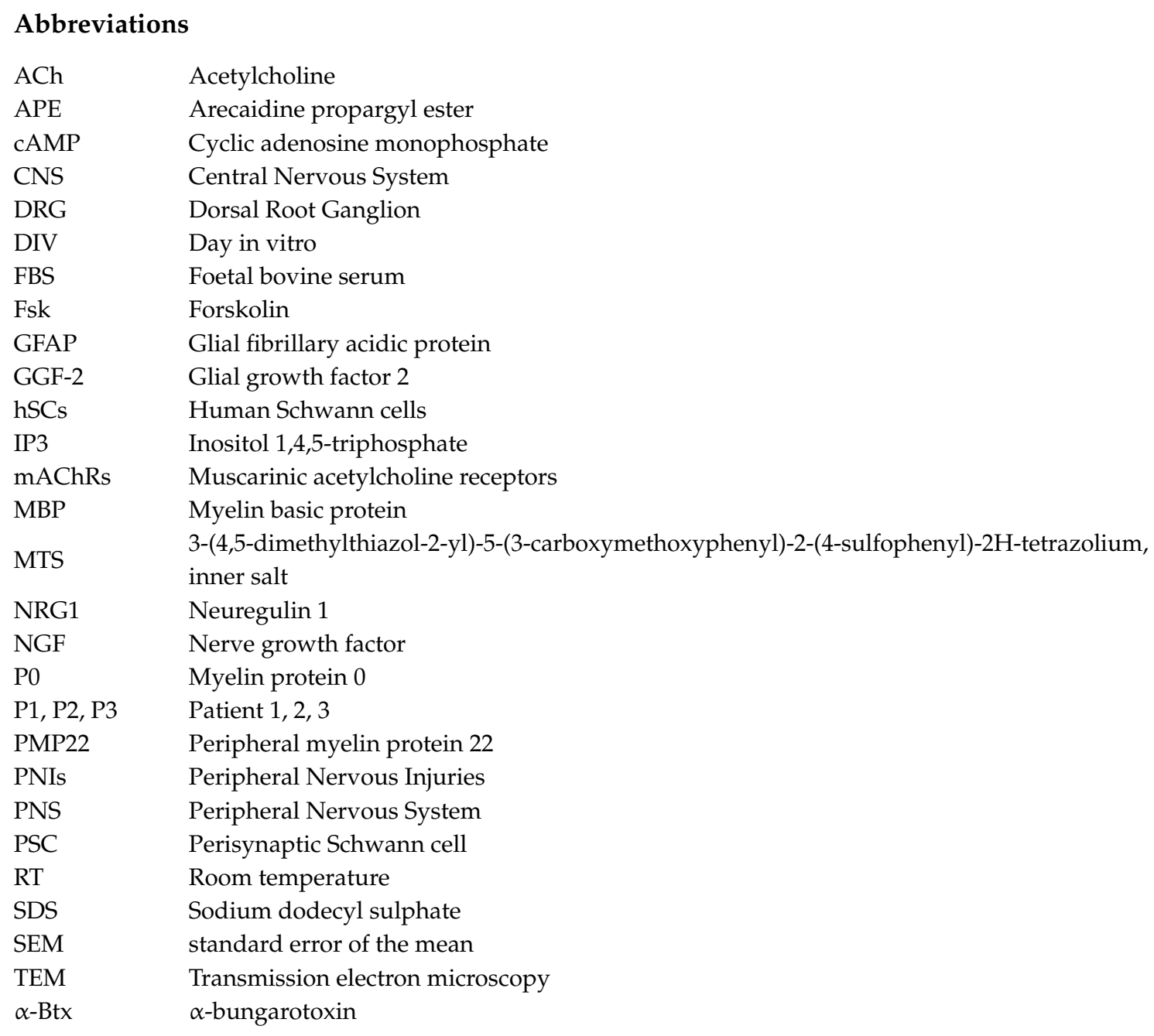

\section{References}

1. Fields, R.D.; Dutta, D.J.; Belgrad, J.; Robnett, M. Cholinergic signaling in myelination. Glia 2017, 65, 687-698. [CrossRef] [PubMed]

2. Fields, R.D. Release of neurotransmitters from glia. Neuron. Glia Biol. 2010, 6, 137-139. [CrossRef] [PubMed]

3. Magnaghi, V.; Procacci, P.; Tata, A.M. Chapter 15 Novel Pharmacological Approaches to Schwann Cells as Neuroprotective Agents for Peripheral Nerve Regeneration, 1st ed.; Elsevier Inc.: Amsterdam, The Netherlands, 2009; Volume 87. [CrossRef] 
4. Tata, A.M. Muscarinic Acetylcholine Receptors: New Potential Therapeutic Targets in Antinociception and in Cancer Therapy. Recent Pat. CNS Drug Discov. 2008, 3, 94-103. [CrossRef]

5. Matera, C.; Tata, A.M. Pharmacological Approaches to Targeting Muscarinic Acetylcholine Receptors. Recent Pat. CNS Drug Discov. 2014, 9, 85-100. [CrossRef] [PubMed]

6. Villegas, J. Cholinergic systems in axon-Schwann cell interactions. Trends Neurosci. 1978, 1, 66-68. [CrossRef]

7. Guizzetti, M.; Costa, P.; Peters, J.; Costa, L.G. Acetylcholine as a mitogen: Muscarinic receptor-mediated proliferation of rat astrocytes and human astrocytoma cells. Eur. J. Pharmacol. 1996, 297, 265-273. [CrossRef]

8. De Angelis, F.; Bernardo, A.; Magnaghi, V.; Minghetti, L.; Tata, A.M. Muscarinic receptor subtypes as potential targets to modulate oligodendrocyte progenitor survival, proliferation, and differentiation. Dev. Neurobiol. 2012, 72, 713-728. [CrossRef]

9. Loreti, S.; Vilaró, M.T.; Visentin, S.; Rees, H.; Levey, A.I.; Tata, A.M. Rat Schwann cells express M1-M4 muscarinic receptor subtypes. J. Neurosci. Res. 2006, 84, 97-105. [CrossRef]

10. Uggenti, C.; De Stefano, M.E.; Costantino, M.; Loreti, S.; Pisano, A.; Avallone, B.; Talora, C.; Magnaghi, V.; Tata, A.M. M2 muscarinic receptor activation regulates schwann cell differentiation and myelin organization. Dev. Neurobiol. 2014, 74, 676-691. [CrossRef]

11. Loreti, S.; Ricordy, R.; De Stefano, M.E.; Augusti-Tocco, G.; Tata, A.M. Acetylcholine inhibits cell cycle progression in rat Schwann cells by activation of the M2 receptor subtype. Neuron. Glia Biol. 2007, 3, 269-279. [CrossRef]

12. Piovesana, R.; Faroni, A.; Taggi, M.; Matera, A.; Soligo, M.; Canipari, R.; Manni, L.; Reid, A.J.; Tata, A.M. Muscarinic receptors modulate Nerve Growth Factor production in rat Schwann-like adipose-derived stem cells and in Schwann cells. Sci. Rep. 2020, 10, 7159. [CrossRef] [PubMed]

13. Lehmann, H.C.; Höke, A. Schwann cells as a therapeutic target for peripheral neuropathies. CNS Neurol. Disord. Drug Targets 2010, 9, 801-806. [CrossRef] [PubMed]

14. Wei, Z.; Fei, Y.; Su, W.; Chen, G. Emerging Role of Schwann Cells in Neuropathic Pain: Receptors, Glial Mediators and Myelination. Front. Cell. Neurosci. 2019, 13, 1-8. [CrossRef] [PubMed]

15. Blakeley, J.O.; Plotkin, S.R. Therapeutic advances for the tumors associated with neurofibromatosis type 1, type 2, and schwannomatosis. Neuro. Oncol. 2016, 18, 624-638. [CrossRef] [PubMed]

16. Alessandrini, F.; Cristofaro, I.; Di Bari, M.; Zasso, J.; Conti, L.; Tata, A.M. The activation of M2 muscarinic receptor inhibits cell growth and survival in human glioblastoma cancer stem cells. Int. Immunopharmacol. 2015, 29, 105-109. [CrossRef]

17. Piovesana, R.; Faroni, A.; Magnaghi, V.; Reid, A.J.; Tata, A.M. M2 receptors activation modulates cell growth, migration and differentiation of rat Schwann-like adipose-derived stem cells. Cell Death Discov. 2019, 5, 92. [CrossRef]

18. Kim, H.S.; Lee, J.; Lee, D.Y.; Kim, Y.D.; Kim, J.Y.; Lim, H.J.; Lim, S.; Cho, Y.S. Schwann Cell Precursors from Human Pluripotent Stem Cells as a Potential Therapeutic Target for Myelin Repair. Stem Cell Rep. 2017, 8, 1714-1726. [CrossRef]

19. Pestronk, A.; Drachman, D. Motor nerve terminal outgrowth and acetylcholine receptors: Inhibition of terminal outgrowth by alpha-bungarotoxin and anti-acetylcholine receptor antibody. J. Neurosci. 1985, 5, 751-758. [CrossRef]

20. Georgiou, J.; Robitaille, R.; Charlton, M.P. Muscarinic Control of Cytoskeleton in Perisynaptic Glia. J. Neurosci. 1999, 19, 3836-3846. [CrossRef]

21. Gomez-Sanchez, J.A.; Pilch, K.S.; van der Lans, M.; Fazal, S.V.; Benito, C.; Wagstaff, L.J.; Mirsky, R.; Jessen, K.R. After nerve injury, lineage tracing shows that myelin and Remak Schwann cells elongate extensively and branch to form repair Schwann cells, which shorten radically on re-myelination. J. Neurosci. 2017, 37, 9086-9099. [CrossRef]

22. Salani, M.; Anelli, T.; Augusti Tocco, G.; Lucarini, E.; Mozzetta, C.; Poiana, G.; Tata, A.; Biagioni, S. Acetylcholine-induced neuronal differentiation: Muscarinic receptor activation regulates EGR-1 and REST expression in neuroblastoma cells. J. Neurochem. 2009, 821-834. [CrossRef] [PubMed]

23. von der Kammer, H.; Mayhaus, M.; Albrecht, C.; Enderich, J.; Wegner, M.; Nitsch, R.M. Muscarinic Acetylcholine Receptors Activate Expression of the Egr Gene Family of Transcription Factors. J. Biol. Chem. 1998, 273, 14538-14544. [CrossRef] [PubMed] 
24. Parkinson, D.B.; Bhaskaran, A.; Arthur-Farraj, P.; Noon, L.A.; Woodhoo, A.; Lloyd, A.C.; Feltri, M.L.; Wrabetz, L.; Behrens, A.; Mirsky, R.; et al. c-Jun is a negative regulator of myelination. J. Cell Biol. 2008, 181, 625-637. [CrossRef]

25. Jakubík, J.; Bačáková, L.; El-Fakahany, E.E.; Tuček, S. Positive Cooperativity of Acetylcholine and Other Agonists with Allosteric Ligands on Muscarinic Acetylcholine Receptors. Mol. Pharmacol. 1997, 52, 172-179. [CrossRef] [PubMed]

26. Moser, U.; Lambrecht, G.; Wagner, M.; Wess, J.; Mutschler, E. Structure-activity relationships of new analogues of arecaidine propargyl ester at muscarinic M1 and M2 receptor subtypes. Br. J. Pharmacol. 1989, 96, 319-324. [CrossRef] [PubMed]

27. Tumiatti, V.; Wehrle, J.; Hildebrandt, C.; Moser, U.; Dannhardt, G.; Mutschler, E.; Lambrecht, G. Muscarinic Properties of Compounds Related to Arecaidine Propargyl Ester. Arzneimittelforschung 2000, 50, 11-15. [CrossRef]

28. De Angelis, F.; Marinelli, S.; Fioretti, B.; Catacuzzeno, L.; Franciolini, F.; Pavone, F.; Maria Tata, A. M2 Receptors Exert Analgesic Action on DRG Sensory Neurons by Negatively Modulating VR1 Activity. J. Cell. Physiol. 2014, 229, 783-790. [CrossRef]

29. Ferretti, M.; Fabbiano, C.; Bari, M.D.; Conte, C.; Castigli, E.; Sciaccaluga, M.; Ponti, D.; Ruggieri, P.; Raco, A.; Ricordy, R.; et al. M2 receptor activation inhibits cell cycle progression and survival in human glioblastoma cells. J. Cell. Mol. Med. 2013, 17, 552-566. [CrossRef]

30. Di Bari, M.; Tombolillo, V.; Conte, C.; Castigli, E.; Sciaccaluga, M.; Iorio, E.; Carpinelli, G.; Ricordy, R.; Fiore, M.; Degrassi, F.; et al. Cytotoxic and genotoxic effects mediated by M2 muscarinic receptor activation in human glioblastoma cells. Neurochem. Int. 2015, 90, 261-270. [CrossRef]

31. Lucianò, A.M.; Mattei, F.; Damo, E.; Panzarini, E.; Dini, L.; Tata, A.M. Effects mediated by M2 muscarinic orthosteric agonist on cell growth in human neuroblastoma cell lines. Pure Appl. Chem. 2019, 91, 1641-1650. [CrossRef]

32. Piovesana, R.; Melfi, S.; Fiore, M.; Magnaghi, V.; Tata, A.M. M2 muscarinic receptor activation inhibits cell proliferation and migration of rat adipose-mesenchymal stem cells. J. Cell. Physiol. 2018, 233, 5348-5360. [CrossRef] [PubMed]

33. Pacini, L.; De Falco, E.; Di Bari, M.; Coccia, A.; Siciliano, C.; Ponti, D.; Pastore, A.L.; Petrozza, V.; Carbone, A.; Tata, A.M.; et al. M2 muscarinic receptors inhibit cell proliferation and migration in urothelial bladder cancer cells. Cancer Biol. Ther. 2014, 15, 1489-1498. [CrossRef] [PubMed] 